\title{
Survey on Shot Boundary Detection Methods
}

\author{
${ }^{1}$ Ms. Hina Shahanur Sayyad, ${ }^{2}$ Prof. Jadhav Dattatray A.
}

${ }^{2}$ Professor, Department of Electronics and Telecommunication, Jspm's Imperial college of engineering and research, Pune, India. ${ }^{1}$ mumtajsayyad143@gmail.com, ${ }^{2}$ prof.jadhavda@gmail.com

Abstract The increase in the growth of multimedia technology leads to an increase in multimedia content in a large amount. Hence it is important to access only interesting video content instead of the whole video. For effective indexing and retrieving the interesting content from the whole video, the Content-Based Video Retrieval (CBVR) is used. Shot boundary detection is one of the most important and necessary steps. It is used to partitioning the video into shots that are necessary for indexing and retrieval of video. Therefore, segmentation plays a significant role in the field of digital image and media processing, computer vision and pattern recognition. In this paper, the recent development for shot boundary detection has been presented.

Keywords-CBVR, Cut, Fade, Machine Learning, shot boundary detection, video processing, Video Retrieval.

\section{INTRODUCTION}

In recent years, digital videos are creating in many fields like hospitals, digital libraries, distance learning, video broadcasting, video on demand, interactive TV, etc. The user searches the data based on text or content-based input. In the case of a text-based video retrieval search system, the videos are annotated using a text keyword. But, the textbased approach is time-consuming also it populates the data with lots of data. The content-based retrieval process gives more correct results than the text-based approach.

Shot boundary detection is important for automatic video indexing. The frame is the basic unit of any video. The basic structure of the video and its components are shown in Fig .1. The frames are indexed by frame number. Video is nothing but the aggregation of the consecutive sequence of frames captured by the camera devices. Video is broken into the same size frames. Ideally, the 25-30 frames per second (fps) are considered for frame extraction. Each frame consist of the scene and each scene consist of shots. In general, it can be said that video can be produced by a combination of shots. the scene may contain one or more shots that describe the story unit within a video.

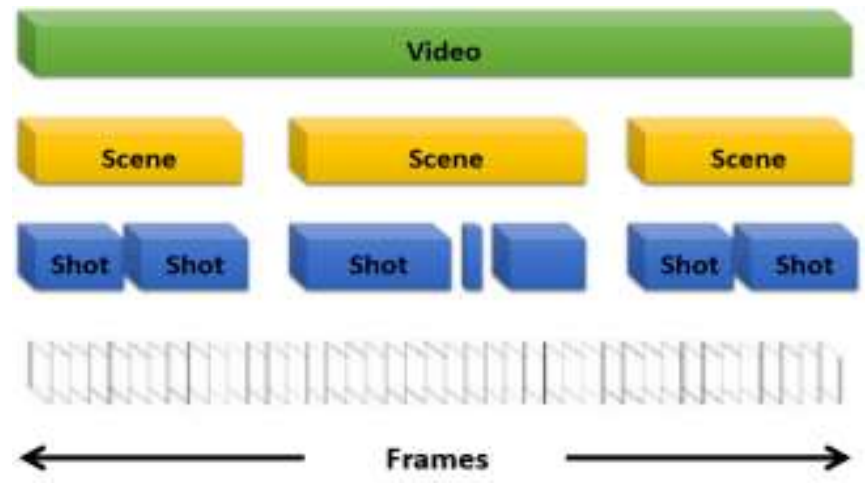

Fig. 1. Structure of video
Shot boundary detection is based on the recognition of visual variation due to the transitions. These variations are generally found while shot change. The shot changes are of two types:

- $\quad$ Abrupt (Hard Cut)

- Gradual (Dissolve, fade-in, fade-out, wipe)

A. Abrupt

Abrupt transition is also called the hard cut is the sudden change in the consecutive frame of the video. A cut is an abrupt shot change that occurs in a single frame.

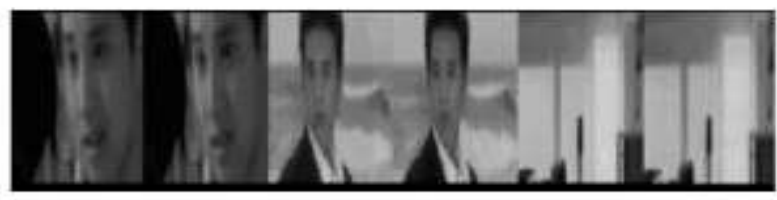

Fig. 1. Example of abrupt or hard cut

\section{B. Gradual}

Gradual changes in the shot boundary detection have occurred over the multiple frames ant it produces the fade in fade out dissolve and wipes. The explanation of the type of each gradual change is as explained below.

1) Fade In Fadeout: It is slow changes in the brightness of the video frame that makes the solid black frame. In the consecutive frame, if the pixels of the first shot are replaced with the pixels of the next shot then wipe occur.

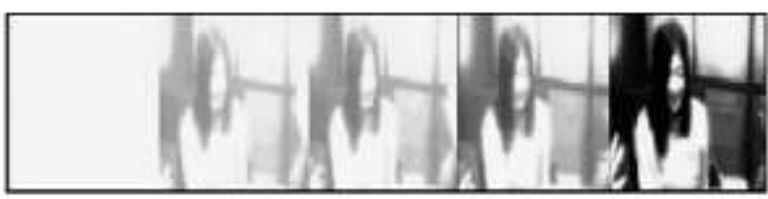

(a) 


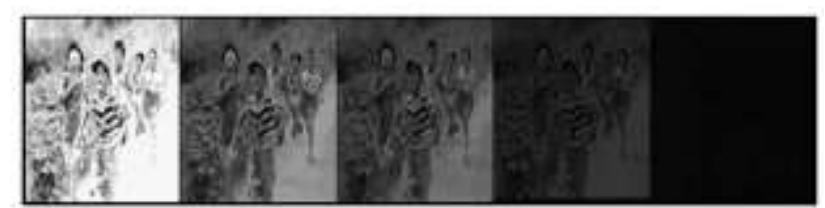

(b)

Fig. 2. Example of Fade transition (a) Fade-in (b) Fade-out

2) Dissolves: In this process, the first shot of the frame getting dimmed and next shot getting brighten then it is illustrating like one image getting superimposed with others.

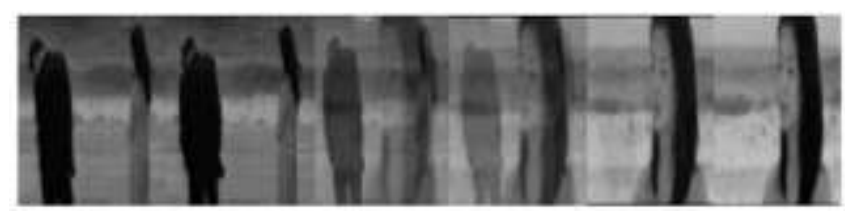

Fig. 3. Example of dissolve gradual transition

\section{SHOt Boundary DeteCTION METHODS}

There are several states of art methods that have been presented by different researchers. Some of them are reviewed in this section.

\section{Twin Comparision Method}

It is the first method to detect the shot boundary detection which classifies the abrupt and gradual changes in the video. This approach is presented by Zhang et al. [1]. In this approach, dual-threshold is applied to the difference of histogram of intensity value to detect the gradual transitions. This method is based on two threshold value, higher threshold (TH) and Lower threshold (TL). TH is used to detect the discontinuity value corresponding to cuts, and TL is applied to the remaining discontinuity values. The gradual changes occur when discontinuity value is greater than the TL value. The summation of consecutive discontinuity values started from that point to the cumulative sum goes beyond higher threshold value TH. The endpoint of the gradual transition set at the last discontinuity value inclusive in the sum.

The primary problem in this approach is that if the threshold value is not set properly then it generates more false-positive values. This approach shows the average precision value is $77.2 \%$ and the recall value is $82 \%$.

\section{Plateau Detection Method}

It is noted that the comparison based is not that effective to detect the gradual transition. Hence to overcome these disadvantages, Yeo and Liu [2] used the difference between the current and $\mathrm{K}^{\text {th }}$ frame. It is represented as,

$$
D_{1} i^{T} k=d\left(x_{1}(i) x_{1}(i+k)\right)
$$

If, the value of k's chosen to be greater than the length of gradual transition, then the sequence $D_{1} i^{T}$ exhibit its plateau max-width. A significant plateau at location $i$ is characterized by a sequence of similar values $D_{j}^{k}, j=1-$ $s, \ldots, 1+s$, which are constantly higher than the successive values. The value of $s$ is directly proportional to the difference between the value of $\mathrm{k}$ and the transition length. This method applies to the linear and nonlinear gradual transitions.

\section{E. Intensity Variance Method}

In this method, the gradual changes are detected by the intensity difference between the consecutive frames or shots in the video. This method is first proposed by Meng et al. [3]. It exploits the DCT-DC coefficients and motion vectors to detect gradual changes. Theoretically, the shape of most dissolves shows parabolic nature. Then the researcher tried to use of width and depth of the curve. However, in actual conditions, the graph is not pronounced due to noised and abrupt motion in the video.

\section{F. Feature-Based Detection}

It is based on measuring the fractional change at edges in the temporal domain. During a cut or dissolve, the edges of the new intensity appear away from the location of existing edges. The edge pixels that emerge or vanish far from existing edge pixels are considered as entering or exiting edge pixel respectively. Cut, fades and dissolve can be detected by the sum of entering and exiting edge pixels while wipes can be detected by their spatial distributions [4].

\section{G. Luminance Histogram Difference Curve Method}

The accuracy of cut detection can be improved by utilizing the adaptive threshold computed from the local window applied to the luminance histogram difference curve. It is developed by Truong et al. [5]. Also, the existing effects were calculated based on the mathematical models for generating ideal fades and dissolves. The constraint on the frame luminance and variance curve was derived to get rid of false positives (FP) caused by camera and object motions during gradual transitions.

\section{H. Double Chromatic Difference (DCD) Method}

Dissolve detection can be detected by using a variance, gradient magnitude, and DCD. The segmentation is the first step of the DCD algorithm where the video is divided into overlapping categories of "potential dissolve" and "Nonpotential dissolve" using edge-based or pixel-based statistics.

The second step of the DCD detector uses segmentation to define the single synthetic dissolve per "potential dissolve segment", beginning and ending at the first and last frame of the segment, respectively. From these frames (starting and ending), the center frame of synthetic dissolve is formed and compared to the intermediate footage. If the shape of the error over time is parabolic shaped, the potential-dissolve segment is accepted. [6] 


\section{Wavelet Transform Method}

The wavelet transform method is presented by Li Yufeng et al. [7] in which wipe transition was considered a vital mode of gradual transition. In this method, each color sub-image and edge sub-image are decomposed using Daubechies 4 (db4) Wavelet transform. The image is decomposed into $8 \times 8$ pixel blocks to minimize the noise influence effectively and the Gaussian model is used to amend the threshold in detecting the potential wipe transition. This method is tested on the video sequences of news, sport, films, MTV and other videos.

This approach is used a Hough line transform to detect the straight lines at the center and the threshold is found dynamically. Therefore this method is reliable to detect the wipe transition reliability in the uncompressed video. In the future, this method can be extended to compressed video as well.

\section{J. Slipped Window Method}

This method is proposed by Tuanfa Qin et al. [8] where the fast shot boundary is detected based on the k-step slipped window by taking a sufficient portion of edit feature and low feature video shots. In this method, the distance of the images of both the ends was calculated and selects candidate fragments that comprise of shot boundaries by an adaptive threshold. The efficiency of this method will be expressed by comparison of calculating amounts and comparison of accuracy. This method is capable of improving the accuracy of shot detection and to minimize the amount of calculation. The parameters, values, and formulae obtained in these approach posses a very high common ability.

\section{K. Temporal Video Segmentation}

Chen Yinzi et al. [9] presented a method for temporal video segmentation based on detection of shot abrupt transition and gradual transition, which takes the circumstances of user terminals into account and produces various kinds of summarization for the individuals. From the results of this system, it is observed that it was obvious that the video summarization could able to convene the browsing necessities of the user which in turn allows the user to yield better browsing experience.

\section{Correlation Metric Method}

Abdul Hameed et al. [10] presented a framework in which a set of a selection of representative keyframes is done which are useful in summarizing the content of the entire video into an abstract for the creation of efficient indexing. Then the videos are classified into its constituent variable size block followed by the selection of better possible similarity metrics. This segmented algorithm is used to detect the shot transition of any type. This method will make use of motion estimation based upon a correlation metric. The results obtained through this approach will prove the effectiveness of the approach.

\section{Constant False Alarm Rate (CFAR) Content-based video retrieval Algorithm}

CFAR method is used for video segmentation and shot change detection [11]. In this algorithm, the theoretical threshold calculation strategy using a non-parameter based processing technique is developed to get the mark of controllable precision as well as an evaluative recall for video cut detection. Simulation results show that this algorithm leads to a very good detection performance of precision of about $90 \%$.

\section{N. Machine Learning Approach}

Machine learning is one of the most popular technology in this decade. There were different approaches developed for shot boundary detection using machine learning. A novel dissolves detection algorithm using machine learning and the multi-resolution concept was proposed by Lienhart R, et al. [12]. This approach is not that concerned about actual features used for dissolve shot detection but concern more about the general framework for gradual transition recognition.

\section{O. Deep Learning Approach}

Ahmed Hassanien et al. [13] present the Spatio-temporal Convolutional Neural Network for the SBD technique. This technique requires a large dataset. Since the dataset is not large enough, this system generated synthetic data by using image compositing models. This algorithm is applied on real and synthetic data containing about 4.7 million frames. This system can detect the dissolve gradual detection and significant improvement in wipe transition. The deep learning-based algorithm presented in this section is 11 times faster than the state of the art method.

In another approach presented by Tomas Soucek et al [14] where the author presents the TransNet neural network based on dilated 3D convolution Neural Network (3DCNN). The architecture of 3DCNN is shown in Fig. 4.

The 3DCNN architecture consists of four $3 \mathrm{D} 3 \times 3 \times 3$ convolution operations. This approach can reduce the trainable parameter significantly. These multiple layered DCNN cells on top of each other followed by max-pooling form a stacked DDCNN block. TransNet contains such multiple Spatial DDCNN blocks. These two fully connected layers refine the features extracted by convolution layers and predict the possible shot boundary for every frame representation. ReLU activation function is used in each layer to increase the non-linearity in the network. This network is 100 times faster than real-time on single powerful GPU2. 


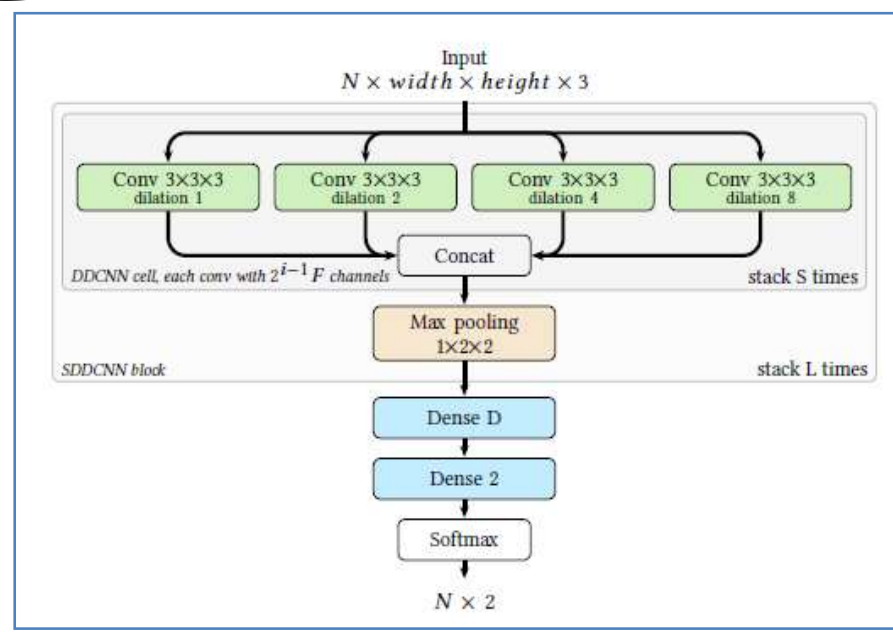

Fig. 4. TransNet shot boundary detection network architecture

Some of the features of the Convolution Neural Network are enlisted below.

1) Standard Neural Networks usually takes an image as an input, CNN takes an image as an input vector of size of an image (height $\mathrm{x}$ width), there is no need to extract the features $\mathrm{CNN}$ itself understand the features.

2) The ordinary neural network does not scale well for a full-sized image (say the size of image 100x100x3), then the network has to process 30000 neurons hence it is extremely costly within the network.

3) It is widely used for image processing, computer vision, and pattern recognition.

4) It has a simple architecture

5) It required very less training parameters

6) It has high flexibility.

7) The weighting structure is analogous to the biological neural network. It reduces the complex nature of the network model and range of weights

8) It is invariant to the displacement, zoom and alternative distortions for 2D graphic images.

9) The full connectedness of these networks helps to get rid of the overfitting problem

\section{P. Directional Empirical Mode Decomposition (DEMD) Approach}

DEMD is generally used in the signal processing algorithms but in this approach the DEMD approach is used to detect the transition during the shots. In this method, each frame of the video is decomposed by the DEMD algorithm and converted into the series of Intrinsic Mode Function (IMF) images. The IMF frame with low frequency is replaced with substitute frames which is composed IMF and residual image. These substitute frames are used to calculate the difference during abrupt illumination changes. In addition to this, the region based object tracking method is applied to avoid the mistaken during object motion. This method adopted remove simple as well as effective motion compensation. As a result this method is no longer sensitive to abrupt changes, motions in the shot and zoom in, zoom out cameras, so that the precision is improved for shot boundary detection.

\section{DISCUSSION}

In recent years, there have been many approaches were reported based on shot boundary detection. Earlier, most of the approaches were concentrated on the abrupt change for shot boundary detection but in recent year the work geared towards the gradual transition for shot boundary detection. Also, it is observed that the detection of gradual changes is a bit difficult than the abrupt change. This is because the cuts were well separated by temporally while gradual changes do not contain any time.

Video shot boundary detection algorithms mostly found the difficulty of finding the shot boundaries in the presence of object motion near camera and illumination variations. furthermore, different shot boundaries may contains different transitions like abrupt temporal or smooth temporal[16].

In recent years, it is also observed that in the advancement in machine learning algorithms, the application of these algorithms is also increased for shot boundary detection.

\section{CONCLUSION}

Shot boundary detection is a very critical task. In this paper, the methods for shot boundary detection have been present. Shot boundary detection is of two types of abrupt transition and gradual transition. Earlier development in the shot boundary detection concentrated on the abrupt change but in recent years the researchers focused on the development of the algorithm for gradual shot boundary detection. In this paper, thirteen methods were review for shot boundary detection. From the review of a different approach, it is observed that most of the earlier algorithms were performed very poorly for unstructured video data. but the recent machine and deep learning-based algorithms perform well for any type of data.

\section{REFERENCES}

[1] Zhang H.J, Kankanhalli A, Smoliar S.W, TanS.Y, "Automatic partitioning of full-motion video". ACM Multimedia Systems 1(1):10-28,1993.

[2] Yeo B.L, Liu B "Rapid scene analysis on compressed video". IEEE Trans Circuits SystemVideo Techno 5(6):533-544, 1995. Song S, Kwon T, Kim W, "Detection of gradual scene changes for parsing of video data". Proc IS\&T/SPIE, vol 3312. pp 404413,1998 
[3] Meng J, Juan Y, Chang S.F, “ Scene change detection in a MPEG compressed video sequence", Proc. SPIE/IS\&T Symp. Electronic Imaging Science and Technology: Digital Video Compression: Algorithms and Technologies, vol 2419. pp 14-25, 1994.

[4] Zabih R, Miller J, Mai K, "A feature-based algorithm for detecting and classifying production effects" Multimedia Syst7(2):119 128,1999

[5] Truong BT, Dorai C, Venkatesh S. “ New enhancements to cut, fade, and dissolve detection processes in video segmentation", ACM International Conference on Multimedia pp 219-227, 2000.

[6] Lu HB, Zhang YJ, Yao YR, "Robust gradual scene change detection", International Conference on Image Processing 3:304-308,1999.

[7] Li Yufeng, Yang Yinghua, Li Guiju, "A Novel Wipe Transition Detection Method Based on Multi-Feature", 2010 Third International Conference on Knowledge Discovery and Data Mining, pp451-454-2/10

[8] Tuanfa Qin, JiayuGu, Huiting Chen \&Zhenhua Tang, "A Fast shot boundary detection based on K-step slipped window”, pp-190- 195IC-NIDC2010.

[9] Chen Yinzi, Wang Kongqiaoet al, “A Temporal Video Segmentation and Summary Generation Method Based on Shots' Abrupt and Gradual Transition Boundary Detecting”, International Conference on Communication Software and Networks, pp978-07695-3961-4/10.

[10] A. Hameed, "A novel framework of shot boundary detection for uncompressed videos," 2009 International Conference on Emerging Technologies, Islamabad, 2009, pp. 274-279.

[11] Liu, T.-Y., Lo, K.-T., Zhang, X.-D., Fengc, J.: A new cut detection algorithm with constant false-alarm ratio for video segmentation. J. Vis. Commun. Image R. 15, 132-144 (2004)

[12] Lienhart R, "Reliable dissolve detection. Storage and Retrieval for Media Database SPIE 4315:219-230, 2001.

[13] A. Hassanien, M. Elgharib, A. Selim, S.-H. Bae, M. Hefeeda, W. Matusik, "Large-scale fast and accurate shot boundary detection through spatio-temporal convolutional neural networks", May 2017, [online] Available: https://arxiv.org/abs/1705.03281.

[14] Soucek, Tomás et al. "TransNet: A deep network for fast detection of common shot transitions." ArXiv abs/1906.03363 (2019).

[15] Lu HB, Zhang YJ, Yao YR, "Robust gradual scene change detection", International Conference on Image Processing 3:304-308,1999.

[16] Dattatraya A. Jadhav, Dr. Parul S. Arora, Dr. Yogesh Kumar Sharma, "REVIEW OF VIDEO SEGMENTATION APPROACH", Journal of
Emerging Technologies and Innovative Research (JETIR) March 2019, Volume 6, Issue 3, pp. 579-586. 